\title{
Simultaneous resolution of the micromagnetic and spin transport equations applied to current-induced domain wall dynamics
}

\author{
M. Sturma, ${ }^{1,2,3,4}$ C. Bellegarde, ${ }^{1,2,3}$ J.-C. Toussaint, ${ }^{1,4}$ and D. Gusakova ${ }^{1,2,3, *}$ \\ ${ }^{1}$ Univ. Grenoble Alpes, INAC-SPINTEC, F-38000 Grenoble, France \\ ${ }^{2}$ CNRS, SPINTEC, F-38000 Grenoble, France \\ ${ }^{3}$ CEA, INAC-SPINTEC, F-38000 Grenoble, France \\ ${ }^{4}$ CNRS, Institut NEEL, F-38000 Grenoble, France
}

(Received 24 March 2016; revised manuscript received 1 July 2016; published 6 September 2016)

\begin{abstract}
In this paper, we use simulations to study current-induced domain wall dynamics by simultaneously resolving the spin transport and micromagnetic equations for a three-dimensional ferromagnetic strip. In contrast to local approaches, which neglect mutual interaction between spins and magnetic moments, our approach recalculates the spin distribution at each time step using the generalized drift diffusion model, which takes the transverse spin absorption phenomenon into account. We quantified the differences between a local approach and treatment based on a self-consistent method by plotting the domain wall velocity as a function of the domain wall width. We also characterized the domain wall velocity and the Walker breakdown condition as a function of the transverse spin absorption length $l_{\perp}$, which plays a crucial role in domain wall dynamics.
\end{abstract}

DOI: 10.1103/PhysRevB.94.104405

\section{INTRODUCTION}

The interaction between itinerant electron spins and nontrivial magnetic textures, such as magnetic domain walls, is of great interest, not only for basic research, but also for storage applications [1], and in the design of logical devices [2]. Current-induced domain wall motion within ferromagnetic materials has been extensively studied both through simulations and experiments (for a review see [3] and references therein). The spin-transfer torque created by the spin-polarized current acts on the magnetic moments and can thus move the domain wall. In general, the spin-transfer effect is nonlocal due to the mutual interaction between magnetic and electron spin subsystems. Whatever the model type or geometry, allowing for feedback from the magnetization dynamics to the electron spin transport and back can be quite tricky and time consuming [4-9]. For this reason, most theoretical studies of domain wall dynamics over the last decade have used local approximations which neglect the mutual interaction between electron spins and magnetic moments. For example, the spin-transfer torque calculated using the drift-diffusive (DD) model $[10,11]$ can be expressed as a function of local magnetization in the case of slowly varying magnetic texture similar to what is described in Thiaville et al. [12]. A local approach considerably simplifies resolution of the magnetization dynamics equation [12-17] and gives access to the qualitative analytical description of the domain wall behavior [18]. However, local approximations are not compatible with systems with high magnetic gradients, and in particular those with short domain walls or vortices. Indeed, among other things, these approximations do not capture how velocity is affected by the domain wall width, as predicted by theory [19]. Moreover, the initial DD model and its equivalents omit the transverse spin absorption resulting from loss of coherence between different directions of the electron propagation. This ballistic phenomenon is related to

\footnotetext{
*daria.gusakova@cea.fr
}

the ferromagnetic band structure and cannot be neglected when investigating current-induced domain wall dynamics. To take transverse spin absorption into account, Petitjean et al. [20] developed the generalized drift diffusion (GDD) model based on the continuous random matrix theory [21,22]. The GDD is a generalization of the Valet-Fert theory [23].

In this paper, we describe a study of three-dimensional (3D) transverse domain wall dynamics based on resolution of the equations for the GDD model [20] and simultaneously the magnetization dynamics equations in a micromagnetic approach [24]. We show that transverse spin absorption, omitted in the simple DD model, plays a crucial role when calculating the domain wall driving force. Because of this crucial role, our results described here are quite different from those reported in previous self-consistent studies based on the DD model [5]. We also determined how domain wall velocity increases with decreasing domain wall width, a phenomenon which was predicted theoretically [19] but is omitted when applying local approximations.

\section{MODEL}

\section{A. Spin transport model}

In this section, we briefly introduce the GDD theory equations [20] implemented in our software. Similar to initial DD theory expressions for the charge current $j_{k}^{c}(\vec{r})$ and the spin current $\mathbf{j}_{k}^{s}(\vec{r})$, our equations include spatial derivatives of the local charge potential $\mu_{c}(\vec{r})$ and the spin chemical potential (or spin accumulation) $\boldsymbol{\mu}(\vec{r})$

$$
\begin{gathered}
j_{k}^{c}=-l \partial_{k} \mu_{c}-P l\left(\mathbf{m} \cdot \partial_{k} \boldsymbol{\mu}\right), \\
\mathbf{j}_{k}^{s}=-l P \partial_{k} \mu_{c} \mathbf{m}-l \partial_{k} \boldsymbol{\mu}-g_{1} l_{*}^{2} l_{L}^{-1}\left(\mathbf{m} \times \partial_{k} \boldsymbol{\mu}\right) \\
+g_{2} l_{*} \mathbf{m} \times\left(\mathbf{m} \times \partial_{k} \boldsymbol{\mu}\right) .
\end{gathered}
$$

Here, the charge and spin currents as well as the charge potential and the spin chemical potential are expressed in energy units. The index $k=x, y, z$ stands for the spatial coordinates 
with spatial derivative $\partial_{k}=\partial / \partial k, P$ for the polarization, $l_{*}$ for the average mean free path, and $l=\left(1-P^{2}\right)^{-1} l_{*}$ [25]. The unit vector $\mathbf{m}(\vec{r})$ points along the local direction of magnetization. The spin current expression [Eq. (2)] contains two supplementary terms compared to simple DD theory. These two terms are proportional to the cross product $\left(\mathbf{m} \times \partial_{k} \boldsymbol{\mu}\right)$. Their algebraic factors $g_{1}=\left[\left(1+l_{*} l_{\perp}^{-1}\right)^{2}+\left(l_{*} l_{L}^{-1}\right)^{2}\right]^{-1}$ and $g_{2}=g_{1}\left(1+l_{*} l_{\perp}^{-1}\right)-\left(1-P^{2}\right)^{-1}$ are a combination of material parameters and characteristic lengths, including the spin coherence length $l_{\perp}$ and the spin precession length $l_{L}$.

In a stationary regime [26], the equations for the charge and the spin current conservation can be written as follows:

$$
\begin{gathered}
\sum_{k} \partial_{k} j_{k}^{c}=0, \\
\sum_{k} \partial_{k} \mathbf{j}_{k}^{s}=-\frac{l_{*}}{l_{\mathrm{sf}}^{2}} \boldsymbol{\mu}-\frac{1}{l_{\perp}}(\mathbf{m} \times \boldsymbol{\mu}) \times \mathbf{m}+\frac{1}{l_{L}}(\mathbf{m} \times \boldsymbol{\mu}) .
\end{gathered}
$$

Here, $l_{\text {sf }}$ corresponds to the spin flip diffusion length. The simple vector product in Eq. (4) divided by $l_{L}$ represents spin precession similar to the initial DD model [11]. The double vector product in Eq. (4) divided by $l_{\perp}$ introduces the absorption of the transverse spin component to the magnetization vector. This parameter was omitted in previous DD theories, although several authors did suggest including a similar phenomenological term [19,27,28] without spin current renormalization. According to the GDD model, the spin transverse component decays approximately over the length scale $l_{\perp}$ and precesses around the magnetization vector over a $l_{L}$ length scale in a nontextured magnet with $\partial_{k} \mathbf{m}=0$.

Both cross product terms in Eq. (4) contribute to the total spin-transfer torque density $\tau$ and introduce the mutual interaction between the magnetization and the conducting electron spins as follows:

$$
\boldsymbol{\tau}=\frac{\hbar}{2 e^{2} l_{*} \rho}\left[\frac{1}{l_{\perp}}(\mathbf{m} \times \boldsymbol{\mu}) \times \mathbf{m}-\frac{1}{l_{L}}(\mathbf{m} \times \boldsymbol{\mu})\right],
$$

where $\hbar$ is the Planck constant, $e$ is the electron charge, and $\rho$ is the resistivity.

\section{B. Magnetization dynamics}

In order to study domain wall dynamics, we completed Eqs. (1)-(5) with the Landau-Lifshitz-Gilbert (LLG) equation in a micromagnetic approach augmented with the spin-transfer torque term

$$
\frac{\partial \mathbf{m}}{\partial t}=-\gamma_{0}\left(\mathbf{m} \times \mathbf{H}_{\mathrm{eff}}\right)+\alpha\left(\mathbf{m} \times \frac{\partial \mathbf{m}}{\partial t}\right)+\frac{2 \mu_{B}}{M_{S} \hbar} \boldsymbol{\tau} .
$$

We used the following notations for the physical parameters and constants: $\gamma_{0}$ for the gyromagnetic ratio, $\mu_{B}$ for the Bohr magneton, $H_{\text {eff }}$ for the effective field (including the exchange and the demagnetizing fields), $\alpha$ for the Gilbert damping factor, and $M_{S}$ for the saturation magnetization.

\section{Self-consistent approach}

In our numerical self-consistent approach, the micromagnetic [Eq. (6)] and spin transport equations [Eqs. (1)-(5)] (a)

(c)

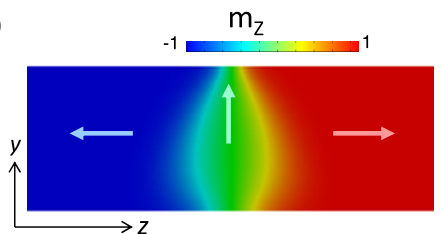

(e)

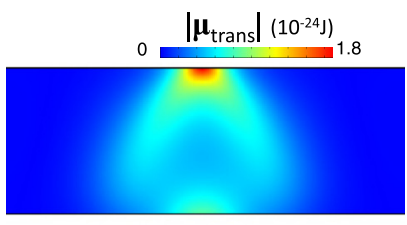

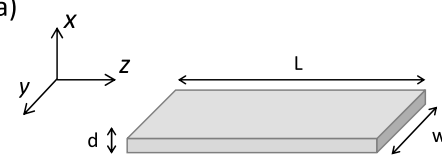

(b)

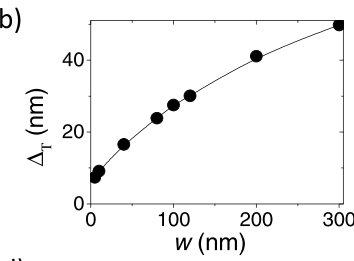

(d)

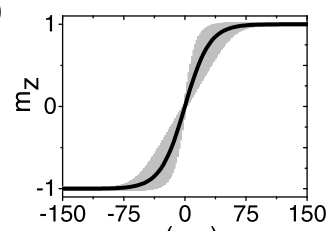

(f)

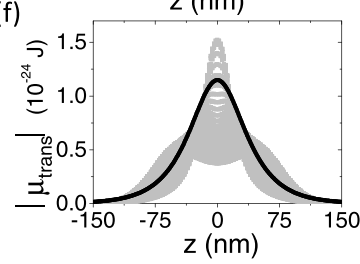

FIG. 1. (a) Geometry studied. (b) Thiele domain wall width as a function of the strip width $w$ for $L=1000 \mathrm{~nm}$ and $d=5 \mathrm{~nm}$. (c) Twodimensional (2D) snapshot of simulated longitudinal magnetization $m_{z}$. (d) Simulated longitudinal magnetization $m_{z}$ as a function of $z$ for $w=120 \mathrm{~nm}$ (gray) and analytical 1D Bloch distribution with $\Delta_{\mathrm{B}}=$ $30 \mathrm{~nm}$ (solid black line). (e) 2D snapshot of simulated transverse spin accumulation amplitude $\left|\boldsymbol{\mu}_{\text {trans }}\right|$. (f) Simulated spin accumulation amplitude $\left|\boldsymbol{\mu}_{\text {trans }}\right|$ as a function of $z$ for $w=120 \mathrm{~nm}$ (gray) and for 1D Bloch distribution with $\Delta_{\mathrm{B}}=30 \mathrm{~nm}$ (solid black line). Graphs (e) and (f) are plotted for $l_{\perp}=1 \mathrm{~nm}, l_{\mathrm{sf}}=5.5 \mathrm{~nm}$ and an applied voltage difference of $0.1 \mathrm{~V}$, which corresponds to an applied current amplitude of $J_{\text {app }}=4 \cdot 10^{11} \mathrm{~A} \mathrm{~m}^{-2}$.

could be resolved simultaneously. At every iteration, the spatial configuration of the magnetic moments takes the electron spin distribution into account, and vice versa. Thus, the spatial distribution of the spin-transfer torque [Eq. (5)] is recalculated from the spin distribution [Eqs. (1)-(4)] for every time step. This distribution can then be injected into a dynamical LLG equation [Eq. (6)] that resets the new spin distribution for the next iteration. Our micromagnetic finite element software feeLLGood [29], supplemented by simple DD theory equations [11], was previously used to study the domain wall dynamics within circular cross-section wires [4]. Several modifications and optimizations were made to this software for this study. For example, we revised the spin transport model to take the ballistic phenomenon into account [20]. We also upgraded the numerical time schema by applying a second-order semiimplicit method with better convergence and stability [30]. In addition, we developed a hexahedron hybrid version of feeLLGood with a regular mesh. This hybrid version combines the advantages of a finite element approach with efficient calculation of the demagnetizing field using fast Fourier transform.

\section{Transverse domain wall}

Figure 1(a) shows the geometry of the rectangular crosssection strip with length $L$, thickness $d$, and width $w$. The longest dimension $L$ corresponds to the $Z$ axis, and the shortest 
dimension $d$ to the $X$ axis. The initial magnetic configuration corresponds to a relaxed transverse tail-to-tail wall located at $z=0$ [Fig. 1(c)]. The magnetic charges at the extremities were numerically removed to prevent magnetization reversal. The spin and charge currents were tangent to the lateral surfaces, and we also used Brown (Neumann) conditions for magnetization components. Spin accumulation tends to zero at the extremities at a distance from strong current and magnetization gradients [Fig. 1(e)]. The micromagnetic parameters used were those of a permalloy material, with $M_{S}=800 \cdot 10^{3} \mathrm{~A} \mathrm{~m}^{-1}, \gamma_{0}=2.21 \cdot 10^{5} \mathrm{~m}(\mathrm{~A} \mathrm{~s})^{-1}, \alpha=0.02$ and the exchange constant $A_{\mathrm{ex}}=1 \cdot 10^{-11} \mathrm{~J} \mathrm{~m}^{-1}$. For the unchanging spin-transport-dependent parameters, we used the following values: $\rho=250 \cdot 10^{-9}(\Omega \mathrm{m})^{-1}, P=0.76, l_{*}=$ $1.72 \mathrm{~nm}, l_{L}=0.7 \mathrm{~nm}$. The strip of $L=1000 \mathrm{~nm}, d=5 \mathrm{~nm}$, and variable $w$ was discretized into $1 \times 2 \times 2 \mathrm{~nm}$ rectangular prisms.

To characterize the distribution of magnetization in all three dimensions, we used the Thiele definition of domain wall width [31,32]

$$
\Delta_{T}=\frac{2 w d}{\int_{\text {volume }}\left(\frac{\partial \mathbf{m}}{\partial z}\right)^{2}} .
$$

The correspondence between the strip width $w$ and $\Delta_{T}$ is shown in Fig. 1(b) for a strip of thickness $d=5 \mathrm{~nm}$. Thus, for example, the domain wall width is estimated to be $\Delta_{T} \approx$ $30 \mathrm{~nm}$ for a strip width of $120 \mathrm{~nm}$. In order to study domain wall velocity as a function of domain wall width (Sec. IV), we varied the strip width $w$ while maintaining a constant strip thickness $d$ and length $L$. Through this approach, we could compare the behavior of a relatively short domain wall to that of a long domain wall with the same material parameters and the same type of transverselike magnetization distribution. Moreover, we also could compare the domain wall behavior in two extreme cases: low spin diffusion with $l_{\mathrm{sf}}<\Delta_{T}$ and high spin diffusion with $l_{\mathrm{sf}}>\Delta_{T}$. The choice to scale the lateral dimension rather than varying the domain wall width by another means does not alter the main results and conclusions presented in this paper. For example, Lee et al. [7] assumed a perpendicularly magnetized nanowire with a variable value for the out-of-plane crystalline anisotropy parameter to vary the domain wall width. In their paper, the domain wall was assumed to be a quasi-one-dimensional (1D) structure, whereas in this paper, we deal with the nontrivial lateral distribution of magnetization, which requires full $3 \mathrm{D}$ micromagnetic calculations.

Figures 1(c) and 1(d) represent the equilibrium spatial distribution of magnetization simulated for $w=120 \mathrm{~nm}$. The spatial distributions corresponding to transverse spin accumulation amplitudes are depicted in Figs. 1(e) and 1(f). As a visual guide, we superimposed simulated distributions (clouds of gray points) on the data obtained with the analytical 1D Bloch distribution (solid line). The magnetization components of the Bloch distribution were expressed as $m_{z}=\tanh \left(z / \Delta_{\mathrm{B}}\right)$ and $m_{y}=\cosh ^{-1}\left(z / \Delta_{\mathrm{B}}\right)$, which includes the domain wall width, $\Delta_{\mathrm{B}}=30 \mathrm{~nm}$. The spin accumulation amplitude is related to the magnetization gradient, and its maximum corresponds to the middle of the domain wall, where the magnetization is most altered. The height of this peak also determines the efficiency of the spin-transfer-torque-related force driving the domain wall.

\section{LONG DOMAIN WALL LIMIT}

Before going into the details of the numerical results obtained with our self-consistent software, we will introduce several useful simplified expressions. In most theoretical and experimental studies of current-induced domain wall displacement, the efficiency of the driving force is characterized by the so-called nonadiabatic parameter $\beta$ [12]. This parameter is introduced as a ratio of the nonadiabatic $\boldsymbol{\tau}_{\text {na }}$ ("out-ofplane" or "fieldlike") spin-transfer torque contribution over the adiabatic $\boldsymbol{\tau}_{a}$ ("in-plane") contribution. In the long domain wall limit, when the electron spins adiabatically follow the magnetization direction, this parameter $\beta$ is assumed to be constant throughout the system. Its value can be calculated by combining the characteristic lengths. For example, the most frequently used local model [12] can be obtained from the simple DD theory [11] for the long domain wall limit $(\Delta \gg$ $\left.l_{\mathrm{sf}}\right)$. In this case, the nonadiabatic parameter $\beta_{\mathrm{NA}}=\left(l_{J} / l_{\mathrm{sf}}\right)^{2}$ is a combination of the two characteristic lengths: the spin flip length $l_{\text {sf }}$ and the exchange length $l_{J}$. In the latter case, the domain wall's driving force does not depend on its width and is determined by the current amplitude applied. Similar to previous theories, the authors of the GDD theory proposed an analytical expression of the nonadiabatic parameter for very long domain walls [20]

$$
\beta_{\text {theor }}=\frac{l_{L} l_{*}}{l_{\mathrm{sf}}^{2}}\left[1+\frac{l_{*} l_{L}^{2}}{l_{\perp} l_{\mathrm{sf}}^{2}}+\left(\frac{l_{L}}{l_{\perp}}\right)^{2}\right]^{-1} .
$$

This parameter correlates with the spin coherence length $l_{\perp}$, as illustrated in Fig. 2(a). In the limit of the long $l_{\perp}$, this expression reduces to $\beta_{\text {theor }} \approx\left(l_{L} l_{*}\right) / l_{\text {sf }}^{2}$, which is similar but not equivalent to the nonadiabatic parameter $\beta_{\mathrm{NA}}=\left(l_{J} / l_{\mathrm{sf}}\right)^{2}$ obtained from the simple DD theory. Indeed, with the long $l_{\perp}$ limit, the double vector product in Eq. (5) disappears to give a spin diffusion equation [Eq. (4)] similar to that obtained with the simple DD theory. Moreover, for the long $l_{\perp}$ length, the $\beta_{\text {theor }}$ parameter tends to saturate towards the permalloy value $\beta_{\mathrm{NA}}=0.04$ with $l_{J}=\sqrt{l_{L} l_{*}}=1.1 \mathrm{~nm}$ and $l_{\mathrm{sf}}=5.5 \mathrm{~nm}$. This result is not surprising since the absorption of the transverse spin component becomes negligible in the long $l_{\perp}$ limit and the system's behavior tends to be dominated by $l_{\text {sf }}$ and $l_{L}$. We will only discuss the similarities between the two models for some limits because solving the complete GDD theory [Eqs. (1)-(5)] never gave the same numerical result as the initial DD theory. At intermediate $l_{\perp}$ values, the spin transverse component absorption phenomenon prevails. In these cases, spin transfer occurs at shorter distances, and thus the nonadiabatic parameter $\beta_{\text {theor }}$ decreases drastically. For example, for a permalloy with $l_{\perp}=1 \mathrm{~nm}$, the nonadiabatic parameter is $\beta_{\text {theor }}=0.026$, which is almost twofold lower than its estimation based on the simple DD theory. Several experimental studies have also reported moderate values of the nonadiabatic parameter comparable to $\alpha$ for long transverse domain walls in permalloy [33,34], and these results are coherent with Eq. (8). 

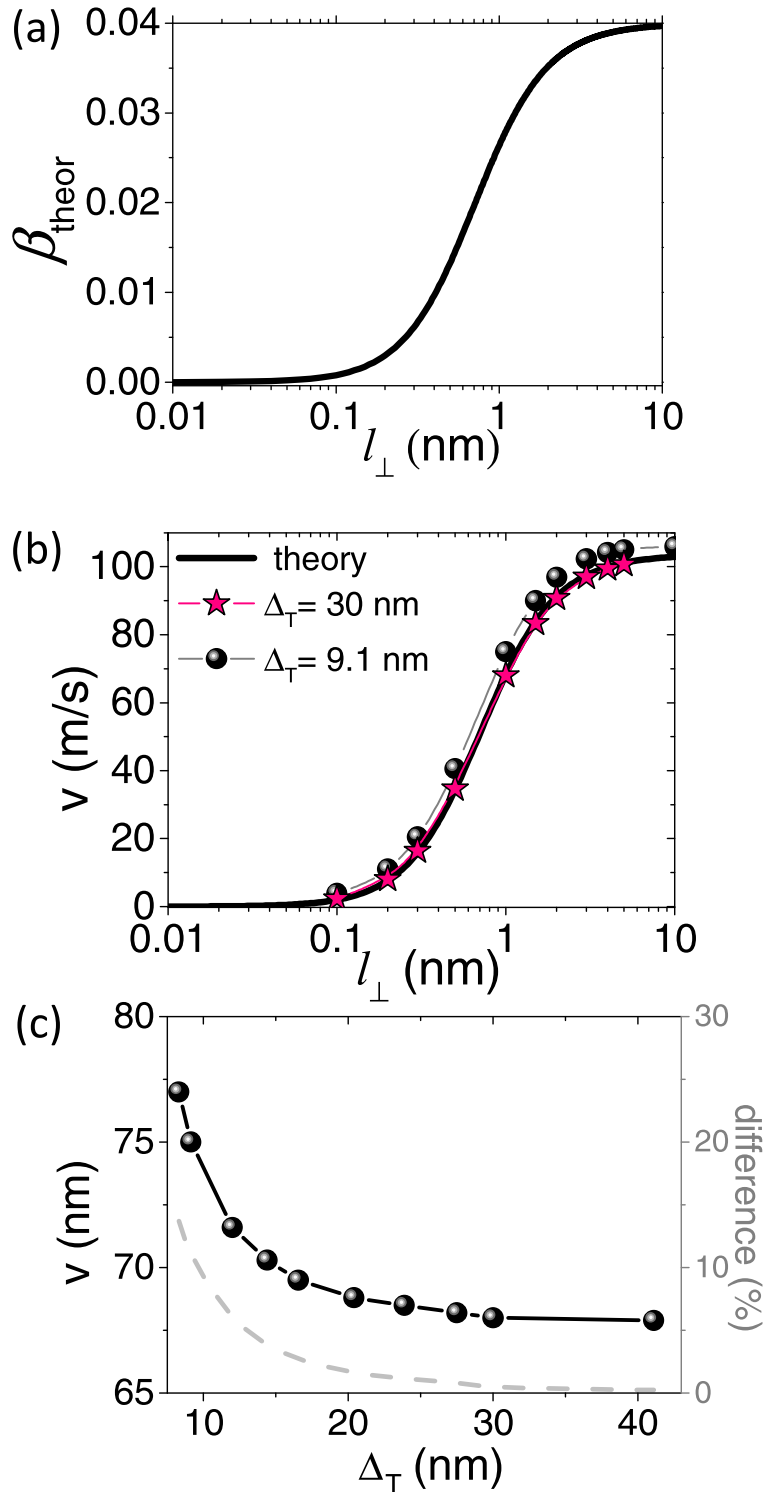

FIG. 2. (a) Analytical value of the nonadiabatic parameter $\beta$ as a function of $l_{\perp}$ according to Eq. (8) with a large domain wall width $\left(\Delta \gg l_{\mathrm{sf}}\right)$. (b) Simulated steady-state velocity $v$ for two values of strip width: $w=120 \mathrm{~nm}\left(\Delta_{T} \approx 30 \mathrm{~nm}\right)$ and $w=10 \mathrm{~nm}$ ( $\left.\Delta_{T} \approx 9.1 \mathrm{~nm}\right)$. The solid black line corresponds to the analytical expression produced by applying Eq. (9). (c) Simulated steady-state velocity and its deviation from the theoretical value calculated using Eq. (9) as a function of the domain wall width for $l_{\perp}=1 \mathrm{~nm}$. Graphs (b) and (c) were plotted for an applied voltage difference of $0.1 \mathrm{~V}$, which corresponds to an applied current amplitude of $J_{\text {app }}=4 \cdot 10^{11} \mathrm{~A} \mathrm{~m}^{-2}$ and $U=52 \mathrm{~m} \mathrm{~s}^{-1}$. (a)-(c) correspond to the spin flip length $l_{\mathrm{sf}}=5.5 \mathrm{~nm}$.

As a result of the reduction in the nonadiabatic parameter with decreasing $l_{\perp}$, the steady-state domain wall velocity should also decrease [Fig. 2(b)]. Indeed, by analogy with the previous models [18], in the long domain wall limit, the steady-state domain wall velocity is expected to correlate linearly with the applied current density. Thus

$$
v_{\text {steady }}=\frac{\beta_{\text {theor }}}{\alpha} U
$$

where

$$
U=\frac{P \mu_{B}}{M_{S} e\left(1-P^{2}\right)} J_{\text {app }},
$$

has the dimension of a velocity and scales with the electrical current density $J_{\text {app }}$. Moreover, critical $U_{\text {Walker values give the }}$ so-called Walker breakdown condition [18]

$$
U_{\text {Walker }}=\alpha M_{S} \frac{\gamma_{0} \Delta}{2|\beta-\alpha|}\left(N_{y}-N_{x}\right),
$$

where $N_{x}$ and $N_{y}$ are the demagnetizing factors along the $x$ and $y$ directions [35]. In these conditions, the domain wall enters a precessional regime with average velocity

$$
\bar{v}=\frac{1+\alpha \beta_{\text {theor }}}{1+\alpha^{2}} U .
$$

The steady-state velocity in Eq. (9), where the nonadiabatic parameter is constant, does not depend on domain wall width. However, this approximation has limits. In the next section, we demonstrate that the spatial dependence of the nonadiabatic parameter, when inherently considered within a self-consistent approach, has a significant impact on current-driven domain wall motion.

\section{NUMERICAL RESULTS}

In this section, we discuss the numerical results obtained using our software. The voltage difference $\Delta V=$ $\left[\mu_{c}(L / 2)-\mu_{c}(-L / 2)\right] / e=J_{\text {app }} \rho L$ applied at the extremities of the strip triggers motion of the domain wall along the $Z$ axis. In this case, the nonadiabatic parameter $\beta$ is no longer constant, and its spatial distribution is nontrivial. The domain wall driving force is determined by its effective value $\beta_{\text {sim }}$, which can be estimated from the velocity data.

\section{A. Velocity vs domain wall width}

We confirmed that the steady-state domain wall velocities obtained numerically differ from those calculated using the simplified analytical formula in Eq. (9) from the previous section. This difference was particularly notable for relatively short domain wall widths and long spin flip lengths. The local spin-transfer torque approach, which neglects the spin diffusion effects, gives lower velocity values. In this case, the spin-transfer torque amplitude at a given point depends only on the local magnetization and its local gradient. In our self-consistent approach, the spin-transfer torque amplitude at a given point is affected by the magnetization and spin distributions over the spin flip length $l_{\text {sf }}$. Thus, the resulting nonadiabaticity is determined to a large extent by the interplay between the domain wall width and the spin flip length values. Figure 3 compares the spatial distribution of the nonadiabatic to adiabatic spin-transfer torque $\left|\boldsymbol{\tau}_{\text {nal }}\right| /\left|\boldsymbol{\tau}_{a}\right|$ ratio for three values of the domain wall width. This quantity approaches a constant value in the long domain wall limit [Fig. 3(c)]. Moreover, it coincides with $\beta_{\text {theor }}$ of the local model. Shorter domain wall widths result in a nontrivial spatial distribution of the $\left|\boldsymbol{\tau}_{\text {na }}\right| /\left|\boldsymbol{\tau}_{a}\right|$ ratio and in higher values of the effective nonadiabaticity [Figs. 3(a) and 3(b)]. High magnetization gradients favor a spin-transfer torque with increased amplitude, resulting in a more nonadiabatic system. Thus, for example, the highest 

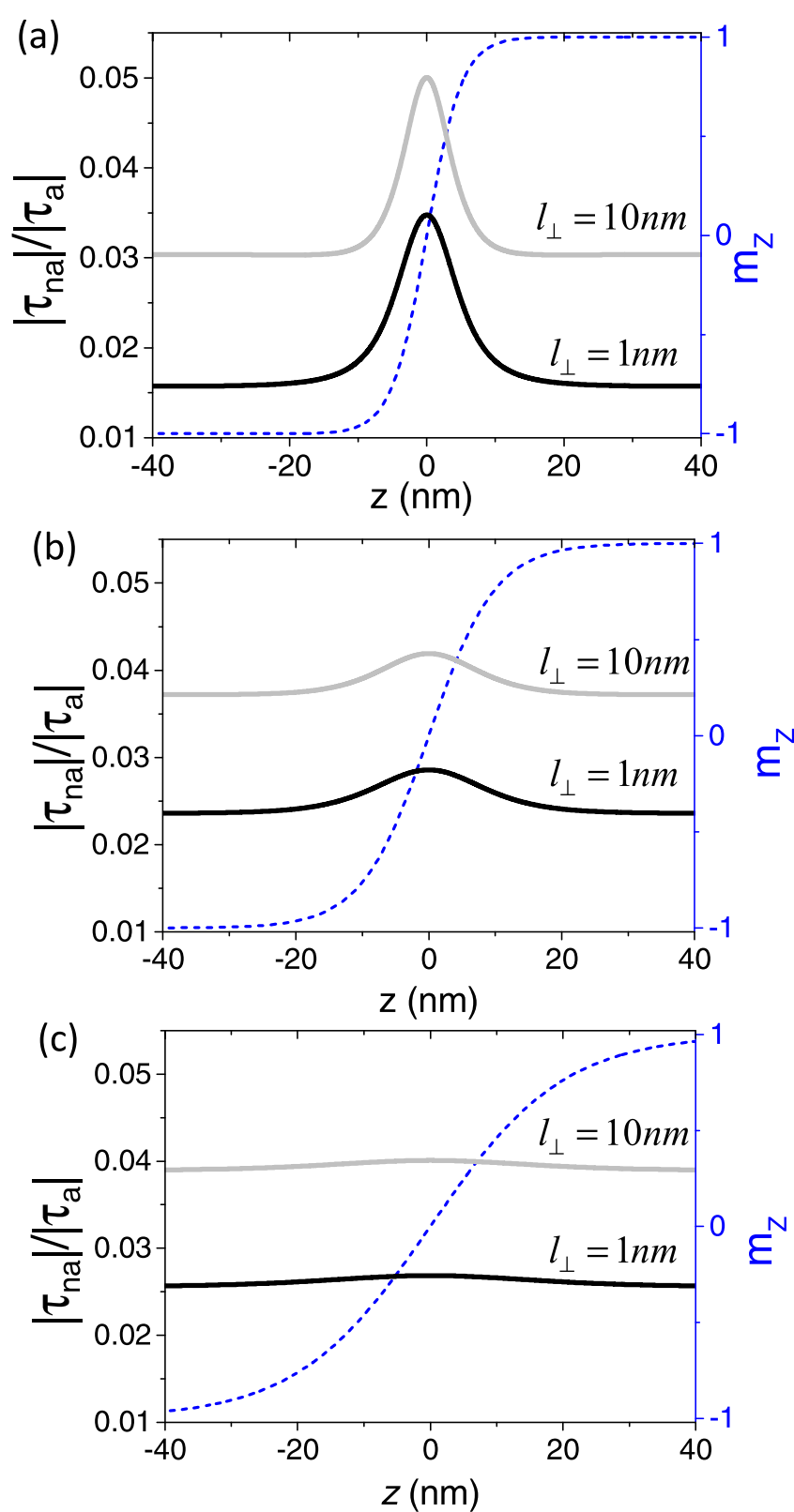

FIG. 3. Ratio of the nonadiabatic spin-transfer torque contribution over the adiabatic contribution as a function of the $Z$ coordinate obtained with the analytical 1D Bloch distribution $m_{z}=\tanh \left(z / \Delta_{B}\right)$ and $m_{y}=\cosh ^{-1}\left(z / \Delta_{B}\right)$. Dashed lines correspond to the longitudinal magnetization spatial distribution. (a) $\Delta_{B}=5 \mathrm{~nm}$. (b) $\Delta_{B}=10 \mathrm{~nm}$. (c) $\Delta_{B}=20 \mathrm{~nm}$.

steady-state velocities are expected for the sharpest magnetic textures, as according to Eq. (9), it is directly proportional to the nonadiabatic parameter. At the same time, the high values of $\beta$ should have less impact on the average velocity in the precessional regime according to Eq. (12).

Figure 2(b) compares the simulated steady-state velocities to those obtained with Eq. (9). As expected, simulated points approach the theoretical curve for a long domain wall width with $l_{\text {sf }} \ll \Delta_{T}$. Nevertheless, the nonlocal velocity was a little higher than the analytical value. The deviation between analytical curve and simulated points was more pronounced for shorter strip widths, and consequently for shorter domain walls. However, the moderate difference in Fig. 2(b) is explained by a very small spin flip length value which does not exceed the domain wall width. Similar qualitative conclusions were reported by Claudio-Gonzalez et al. [5]. These authors simulated velocities self-consistently for long transverse and vortex domain walls using the simple DD model. For both types of domain walls, the nonlocal velocity was higher than the analytical value. In Fig. 2(c), we have plotted the steady-state velocities as a function of the domain wall width. As expected, we observed a significant increase in velocity for short domain walls with high magnetization gradients. A similar relationship between velocity and magnetic gradient was predicted theoretically for a $1 \mathrm{D}$ Bloch wall in the frame of the simple DD theory [19]. In their article, Akosa et al. [19] found the velocity to scale with the inverse square of the domain wall width. Similarly, by applying a semiclassical approach [36], Lee et al. [7] also reported that an increase in effective nonadiabaticity generated high domain

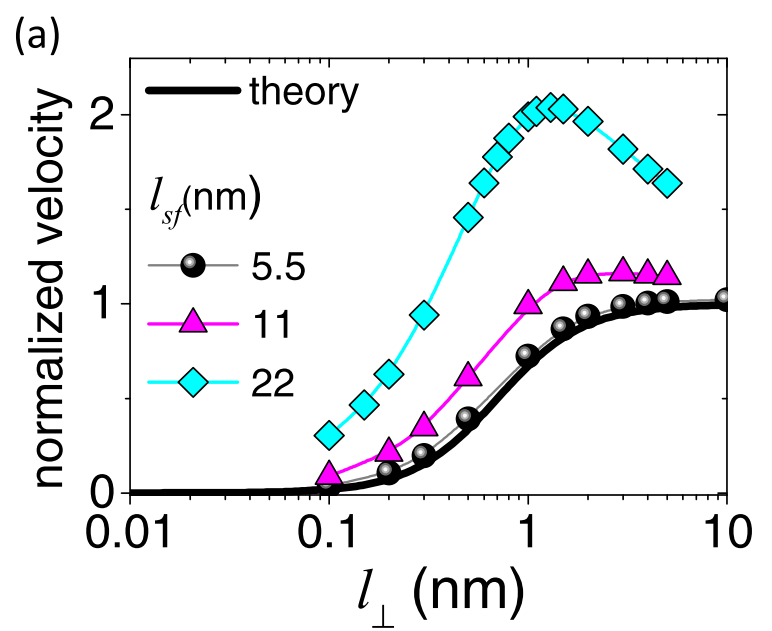

(b)

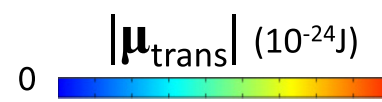

3.2

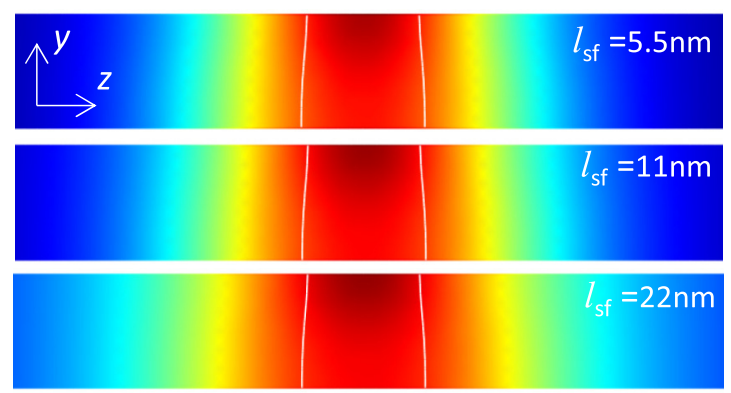

FIG. 4. (a) Simulated steady-state velocity as a function of $l_{\perp}$ for different spin flip lengths $l_{\mathrm{sf}}$. Each curve is normalized by $v_{\text {steady }}$ with the corresponding value of $\beta_{\text {theor }}^{l_{\perp} \rightarrow \infty}$. The solid black line corresponds to the analytical expression produced by applying Eqs. (8) and (9). (b) 2D snapshots of simulated transverse spin accumulation amplitude $\left|\mu_{\text {trans }}\right|$ for three values of $l_{\mathrm{sf}}$ and $l_{\perp}=1 \mathrm{~nm}$. White lines correspond to the longitudinal magnetization isovalues: $m_{z}= \pm 0.5$. Graphs (a) and (b) were plotted for $w=10 \mathrm{~nm}\left(\Delta_{T} \approx 9.1 \mathrm{~nm}\right)$ and for an applied voltage difference of $0.1 \mathrm{~V}$, which corresponds to an applied current amplitude of $J_{\text {app }}=4 \cdot 10^{11} \mathrm{~A} \mathrm{~m}^{-2}$ and $U=52 \mathrm{~m} \mathrm{~s}^{-1}$. 
wall velocities within semi-1D nanowires with very narrow domain walls $(\Delta<2.71 \mathrm{~nm})$.

\section{B. Velocity vs spin flip length}

We observed that high spin diffusion, for which $l_{\mathrm{sf}}>\Delta_{T}$, does not only result in the velocity increase compared to the Eq. (9), but also may change its behavior qualitatively. Figure 4(a) compares the steady-state velocities as a function of $l_{\perp}$ for three values of $l_{\mathrm{sf}}$. Whereas the difference between the simulated points and the analytical curve is moderate for the small $l_{\mathrm{sf}}$ value, it becomes more pronounced with rising $l_{\text {sf }}$. The latter is not captured by the Eq. (9). In contrast to the local approximation, here, the spin distribution at a given point is affected by its environment over the spin flip length. The spin accumulation amplitude decays from its maximum in the center of the domain wall over $l_{\mathrm{sf}}$ distance. Long $l_{\mathrm{sf}}$ length favors a large spatial overlap (a)
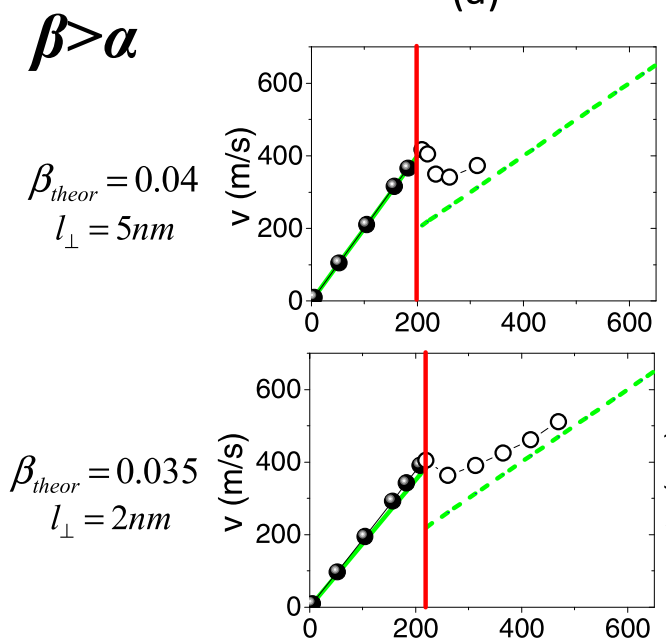

$\begin{aligned} \beta_{\text {theor }} & =0.026 \\ l_{\perp} & =1 \mathrm{~nm}\end{aligned}$

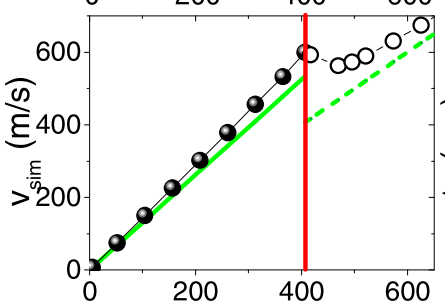

(b)
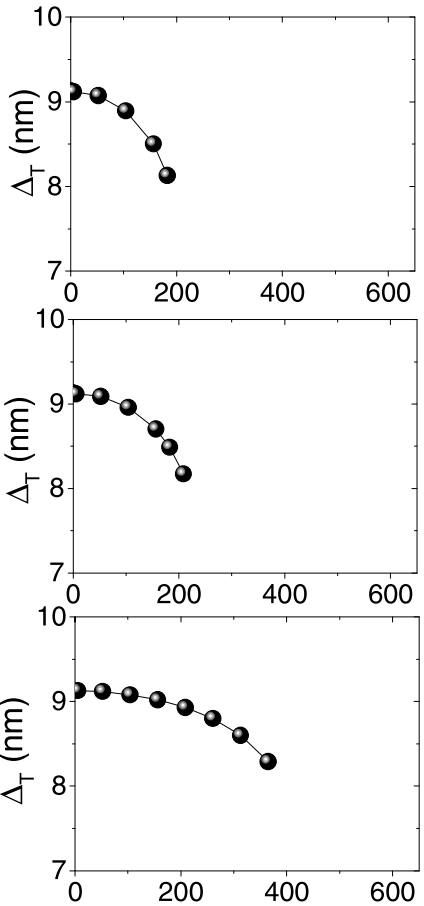

(c)
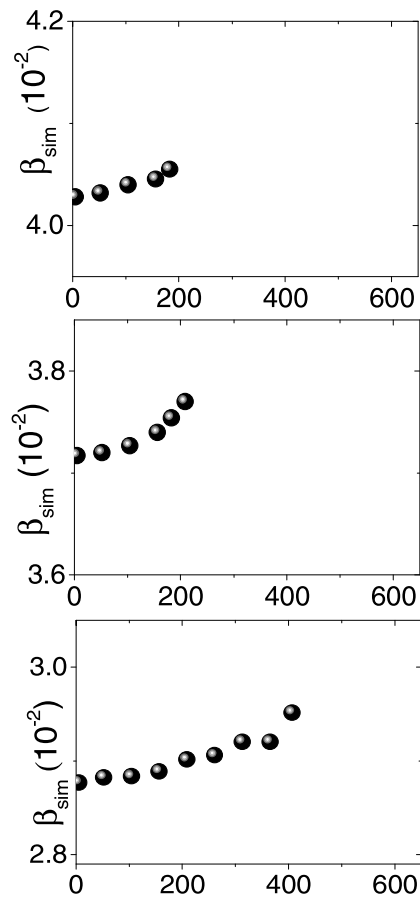
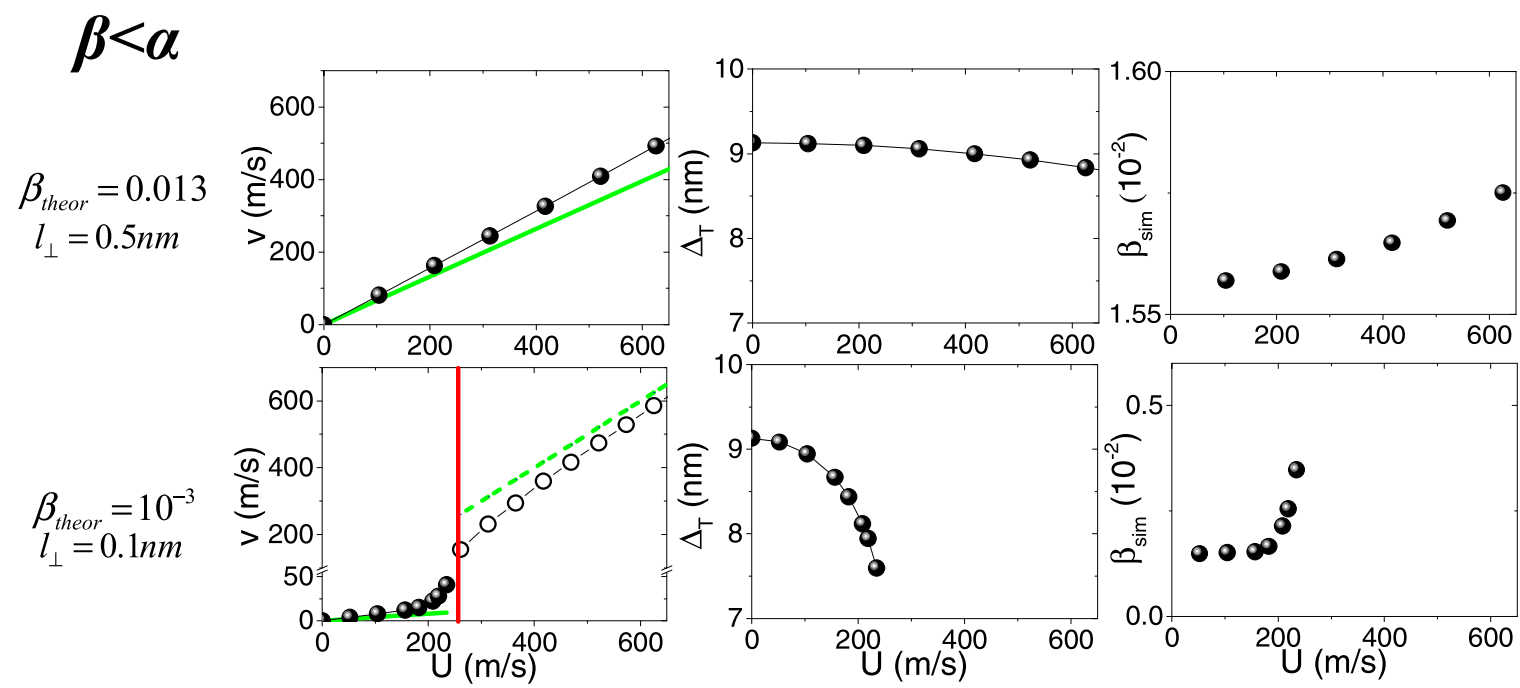

FIG. 5. (a) Simulated velocity $v$ as a function of the applied local current $U$ [Eq. (10)] for different values of $l_{\perp}$ and with $w=10 \mathrm{~nm}$ and $l_{\mathrm{sf}}=5.5 \mathrm{~nm}$. Filled symbols represent the steady-state regime and open symbols the precessional regime. Solid green lines correspond to the steady-state motion velocity according to Eq. (9). Dashed green lines correspond to the average velocity in the precessional regime according to Eq. (12). Vertical red lines indicate the Walker breakdown. (b) Domain wall width $\Delta_{T}^{t \rightarrow \infty}$ as a function of the local current $U$ applied in the steady-state regime. The equilibrium value $\Delta_{T} \approx 9.1 \mathrm{~nm}$ corresponds to $U=0$. (c) The nonadiabatic parameter $\beta_{\text {sim }}$ estimated from simulations as a function of the local current $U$ applied in the steady-state regime. 
between the magnetization gradient and the spin accumulation distribution [Fig. 4(b)]. Thus, the mutual interaction between two subsystems is more efficient, which gives rise to a high effective nonadiabaticity. This explains a noticeable deviation between the analytical curve and the simulated points in Fig. 4(a) for $l_{\mathrm{sf}}>\Delta_{T}$. The curve shape modification may be explained by the complex interplay between the phenomena involved. Accurate description of this behavior requires the self-consistent simulations.

\section{Velocity vs current}

Renormalization of the nonadiabatic parameter results in changes to the velocity versus applied current behavior. Figure 5(a) compares the simulated velocity to the velocities calculated by applying Eqs. (9) and (12) for different values of the spin coherence length $l_{\perp}$. Depending on the $l_{\perp}$ value, for the same material parameters, two distinct cases are possible: $\beta>\alpha$ with $\operatorname{slope}\left(v_{\text {steady }}\right)>\operatorname{slope}(\bar{v})$ and $\beta<\alpha$ with slope $\left(v_{\text {steady }}\right)<\operatorname{slope}(\bar{v})$. In our calculations, both cases were observed. The simulated values exceeded the analytical ones due to the higher nonadiabaticity considered by the self-consistent treatment.

We also noted in our simulations that the steady-state velocity displayed not-quite linear behavior. Thus, the extracted nonadiabatic parameter $\beta_{\text {sim }}$ (as a proportionality coefficient between the current amplitude and the velocity) increased with the current amplitude applied [Fig. 5(c)]. As expected, the deviation of the steady-state velocity from the linear behavior is more pronounced for the long spin flip lengths [Fig. 6]. The deviation from linearity coincided with the decrease in domain wall width related to its structure deformation. The reduction of the domain wall width [Fig. 7(a)] was accompanied with the out-of-plane tilt of the domain wall induced by the rising current amplitude [Fig. 7(b)]. This behavior simulated within the self-consistent approach is qualitatively similar to that obtained using the micromagnetic resolution of the local model [12]. Nevertheless, Fig. 7(a) evidences the discrepancy between two approaches. Indeed, high values of the effective nonadiabatic parameter $\beta$ in the self-consistent approach resulted in a high efficiency of the applied current. In this case, the reduction of the domain wall width occurred for lower applied current values. From purely micromagnetic point of view, we did not observe any significant qualitative changes of the internal domain wall structure between the two approaches. All differences in the steady-state domain wall velocities we attribute to the renormalization of the effective nonadiabatic parameter which depends on the domain wall width to the spin flip length ratio.

The deviation of the steady-state velocity from linearity may be explained by the continuous renormalization of the nonadiabatic parameter $\beta_{\text {sim }}$ with changing domain wall width for each value of the applied current. This mutual interaction between magnetic and spin subsystems was enhanced for larger values of the spin flip length. In contrast to that, in the case of the local model [12], the domain wall width reduction did not affect the velocity versus applied current linear behavior [Fig. 6(a)]. The deviation from linearity observed here may be compared qualitatively to the velocity versus applied magnetic field behavior $[37,38]$. In that case,
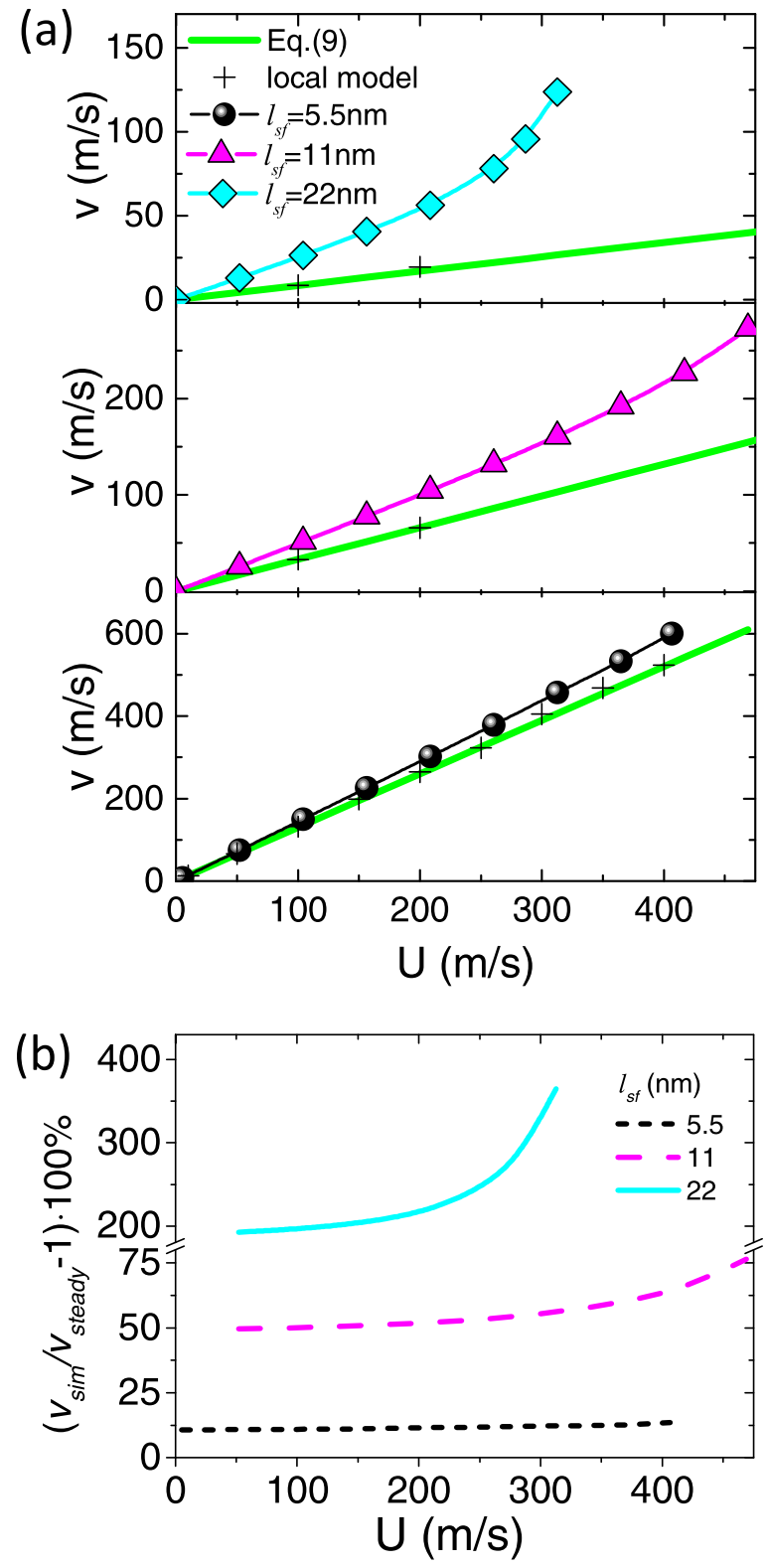

FIG. 6. (a) Simulated steady-state velocity as a function of the applied local current $U$ [Eq. (10)] for different values of $l_{\mathrm{sf}}$. Solid green lines correspond to the steady-state motion velocity according to Eq. (9). Cross symbols correspond to the velocity values obtained with the simplified local micromagnetic model [12]. (b) Deviation of the simulated steady-state velocity from the theoretical value calculated using Eq. (9) as a function of the applied local current for different values of $l_{\mathrm{sf}}$. Graphs (a) and (b) were plotted for $w=10 \mathrm{~nm}$ $\left(\Delta_{T} \approx 9.1 \mathrm{~nm}\right)$.

the velocity is proportional to the domain wall width. Thus, the domain wall width reduction due to the magnetization out-of-plane tilt resulted in a simulated velocity decrease compared to the analytical value.

\section{Walker breakdown}

The threshold for applied current density delimiting the steady-state and precessional regimes is indicated by the vertical red line in Fig. 5(a). Figure 7(c) compares the 

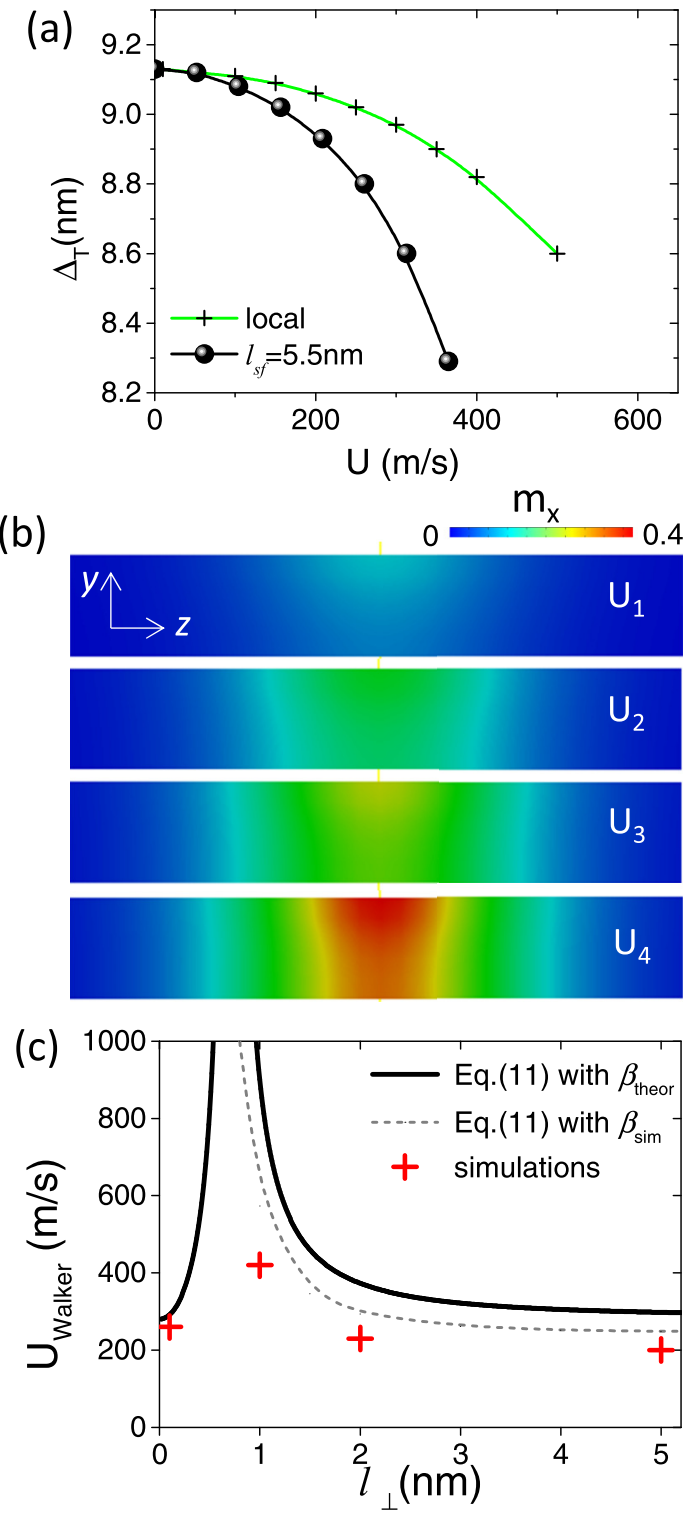

FIG. 7. (a) Steady-state domain wall width as a function of the applied local current $U$ [Eq. (10)]. (b) Spatial distribution of the out-of-plane magnetization component $m_{x}$ for several values of the applied local current: $U_{1}=50 \mathrm{~m} / \mathrm{s}, U_{2}=100 \mathrm{~m} / \mathrm{s}, \quad U_{3}=$ $150 \mathrm{~m} / \mathrm{s}, U_{4}=200 \mathrm{~m} / \mathrm{s}$. (c) Walker breakdown as a function of the spin coherence length $l_{\perp}$. The solid black line corresponds to the result of Eq. (11) with $\beta_{\text {theor }}$ obtained by applying Eq. (8). The dashed line corresponds to the result of Eq. (11) where the nonadiabatic parameter $\beta_{\text {sim }}$ was estimated from the data in Fig. 2(b). Symbols correspond to the observed changes to domain wall motion characterizing the Walker breakdown. All plots correspond to a strip with $w=$ $10 \mathrm{~nm}\left(\Delta_{T} \approx 9.1 \mathrm{~nm}\right)$ and $l_{\mathrm{sf}}=5.5 \mathrm{~nm}$. (a) and (b) correspond to $l_{\perp}=1 \mathrm{~nm}$.

simulated and analytical values of the Walker breakdown as a function of the spin coherence length $l_{\perp}$. The solid black line corresponds to the solution of Eq. (11) with the theoretical value of $\beta_{\text {theor }}$ obtained by applying Eq. (8). This curve reveals that, for a permalloy material with $l_{\perp} \approx 1 \mathrm{~nm}$, Walker breakdown would occur at very high current values.
This effect will be beneficial in potential applications. As indicated above, the simulated parameter $\beta_{\text {sim }}$ was greater than $\beta_{\text {theor }}$ for all values of $l_{\perp}$. Consequently, the $U_{\text {Walker }}$ dependence calculated using Eq. (11) with $\beta_{\text {sim }}$ [dashed line in Fig. 7(a)] shifts to the left, towards smaller values of $l_{\perp}$. The symbols in Fig. 7(a) correspond to the alteration to domain wall motion characteristic of the Walker transition observed in simulations: from laminar motion with constant velocity to forward drift with oscillating domain wall width and velocity. As expected, these symbols fit well with the dashed line.

\section{CONCLUSION}

This paper presents a study of the transverse domain wall dynamics based on simultaneous resolution of the spin transport and micromagnetic equations for a 3D ferromagnetic strip. We observed considerable differences between the simplified and self-consistent treatments of the current-induced domain wall dynamics. These differences clearly highlighted the limitations of a local spin-transfer torque approach, which neglects spin diffusion effects and thus underestimates domain wall velocities. Moreover, while a local approach is sufficient with long domain wall limit, it does not hold true in many situations requiring self-consistent treatment of the spin and magnetization dynamics. We reported both qualitative and quantitative modifications of the domain wall velocity due to the spin diffusion phenomena. In contrast to the local approximation, where the domain wall velocity does not depend on its width, the simultaneous resolution of the spin transport and micromagnetic equations indicated an increase in domain wall velocity with decreasing domain wall width. Thus, due to spin diffusion effects, the same applied current density can move shorter domain walls or other sharp magnetic textures (vortices, Bloch points, etc.) much more effectively. In addition, high spin diffusion with long spin flip length $l_{\text {sf }}$ results in qualitative changes of velocity versus current behavior compared to theoretical predictions. Furthermore, we characterized domain wall velocity and the Walker breakdown condition as a function of the transverse spin absorption length $l_{\perp}$. This analysis revealed that the transverse spin absorption, omitted in the simple DD model, plays a crucial role when calculating the domain wall driving force and its velocity. As a consequence, the domain wall velocity values presented here were quite different to those presented in studies based on the simple DD model. Indeed, the transverse spin absorption phenomenon involves an additional characteristic length $l_{\perp}$, which dominates the spin accumulation distribution in the system, and thus the efficiency of spin-transfer torque. For very short $l_{\perp}$, drastic attenuation of transverse spin accumulation could even result in an overall decrease in spin-transfer torque efficiency. This effect should be taken into consideration when performing current-induced domain wall dynamics experiments.

\section{ACKNOWLEDGMENT}

This paper was funded by the Alpes Grenoble Innovation Recherche AGI14SMI15 project. 
[1] S. Parkin, M. Hayashi, and L. Thomas, Science 320, 190 (2008).

[2] D. Allwood, G. Xiong, C. Faulkner, D. Atkinson, D. Petit, and R. Cowburn, Science 309, 1688 (2005).

[3] O. Boulle, G. Malinowski, and M. Kläui, Mater. Sci. Eng. R 72, 159 (2011).

[4] M. Sturma, J.-C. Toussaint, and D. Gusakova, J. Appl. Phys. 117, 243901 (2015).

[5] D. Claudio-Gonzalez, A. Thiaville, and J. Miltat, Phys. Rev. Lett. 108, 227208 (2012).

[6] C. Abert, M. Ruggeri, F. Bruckner, C. Vogler, G. Hrkac, D. Praetirius, and D. Suess, Sci. Rep. 5, 14855 (2015).

[7] K.-J. Lee, M. D. Stiles, H.-W. Lee, J.-H. Moon, K.-W. Kim, and S.-W. Lee, Phys. Rep. 531, 89 (2013).

[8] K. Matsushita, J. Sato, and H. Imamura, J. Appl. Phys. 105, 07D525 (2009).

[9] J.-i. Ohe and B. Kramer, Phys. Rev. Lett. 96, 027204 (2006).

[10] S. Zhang, P. M. Levy, and A. Fert, Phys. Rev. Lett. 88, 236601 (2002).

[11] S. Zhang and Z. Li, Phys. Rev. Lett. 93, 127204 (2004).

[12] A. Thiaville, Y. Nakatani, J. Miltat, and S. Suzuki, Europhys. Lett. 69, 990 (2005).

[13] J. He, Z. Li, and S. Zhang, J. Appl. Phys. 99, 08G509 (2006).

[14] S. Fukami, T. Suzuki, N. Ohshima, K. Nagahara, and N. Ishiwata, J. Appl. Phys. 103, $07 E 718$ (2008).

[15] E. Martinez, L. Lopez-Diaz, O. Alejos, L. Torres, and M. Carpentieri, Phys. Rev. B 79, 094430 (2009).

[16] H. Szambolics, J. C. Toussaint, A. Marty, I. M. Miron, and L. D. Buda-Prejbeanu, J. Magn. Magn. Mater. 321, 1912 (2009).

[17] M. D. Mascaro and C. A. Ross, Phys. Rev. B 82, 214411 (2010).

[18] A. Mougin, M. Cormier, J. P. Adam, P. Metaxas, and J. Ferré, Europhys. Lett. 78, 57007 (2007).

[19] C. A. Akosa, W.-S. Kim, A. Bisig, M. Klaui, K.-J. Lee, and A. Manchon, Phys. Rev. B 91, 094411 (2015).

[20] C. Petitjean, D. Luc, and X. Waintal, Phys. Rev. Lett. 109, 117204 (2012).

[21] V. S. Rychkov, S. Borlenghi, H. Jaffres, A. Fert, and X. Waintal, Phys. Rev. Lett. 103, 066602 (2009).

[22] S. Borlenghi, V. Rychkov, C. Petitjean, and X. Waintal, Phys. Rev. B 84, 035412 (2011).
[23] T. Valet and A. Fert, Phys. Rev. B 48, 7099 (1993).

[24] A. Hubert and R. Schäfer, Magnetic Domains (Springer Verlag, Berlin, 1998).

[25] In Ref. [20], the polarization $P$ and the average mean free path $l_{*}$ are defined as $P=\left(l_{\uparrow}-l_{\downarrow}\right) /\left(l_{\uparrow}+l_{\downarrow}\right)$ and $1 / l_{*} \equiv 1 / l_{\uparrow}+1 / l_{\downarrow}$ with $l_{\uparrow(\downarrow)}$ the mean free path for majority (minority) electrons. These parameters are equivalent to the usual ones of Valet-Fert theory $[21,23]$.

[26] In stationary regime, the time derivatives of the local charge potential $\partial_{t} \mu_{c}$ and of the spin chemical potential $\partial_{t} \boldsymbol{\mu}$ are neglected in comparison to that of magnetic moments $\partial_{t} \mathbf{m}$. Both transport variables $\mu_{c}$ and $\boldsymbol{\mu}$ are treated within the long time limit since their characteristic time scales several orders of magnitude shorter than that of the magnetic moments.

[27] P. M. Haney, H.-W. Lee, K.-J. Lee, A. Manchon, and M. D. Stiles, Phys. Rev. B 87, 174411 (2013).

[28] P. Chureemart, I. D'Amico, and R. W. Chantrell, J. Phys.: Condens. Matter 27, 146004 (2015).

[29] E. Kritsikis, A. Vaysset, L. D. Buda-Prejbeanu, F. Alouges, and J.-C. Toussaint, J. Comput. Phys. 256, 357 (2014).

[30] F. Alouge, E. Kritsikis, J. Steiner, and J.-C. Toussaint, Numer. Math. 128, 407 (2014).

[31] A. A. Thiele, Phys. Rev. Lett. 30, 230 (1973).

[32] A. Thiaville, Y. Nakatani, F. Piechon, J.Miltat, and T. Ono, Eur. Phys. J. B 60, 15 (2007).

[33] M. Hayashi, L. Thomas, Ya. B. Bazaliy, C. Rettner, R. Moriya, X. Jiang, and S. S. P. Parkin, Phys. Rev. Lett. 96, 197207 (2006).

[34] M. Eltschka, M. Wotzel, J. Rhensius, S. Krzyk, U. Nowak, M. Klaui, T. Kasama, R. E. Dunin-Borkowski, L. J. Heyderman, H. J. van Driel, and R. A. Duine, Phys. Rev. Lett. 105, 056601 (2010).

[35] A. Aharoni, J. Appl. Phys. 83, 3432 (1998).

[36] Jiang Xiao, A. Zangwill, and M. D. Stiles, Phys. Rev. B 73, 054428 (2006).

[37] Y. Nakatani, A. Thiaville, and J. Miltat, Nat. Mater. 2, 521 (2003).

[38] E. Martinez, L. Lopez-Diaz, L. Torres, C. Tristan, and O. Alejos, Phys. Rev. B 75, 174409 (2007). 